\title{
A STUBBORNLY LARGE MASS OF COLD DUST IN THE EJECTA OF SUPERNOVA 1987A*
}

\author{
M. Matsuura $^{1}$, E. DweK ${ }^{2}$, M. J. Barlow ${ }^{1}$, B. Babler ${ }^{3}$, M. Baes $^{4}$, M. Meixner $^{5}$, José Cernicharo $^{6}$, Geoff C. Clayton ${ }^{7}$, \\ L. Dunne ${ }^{8,9}$, C. Fransson ${ }^{10}$, Jacopo Fritz $^{4}$, Walter Gear ${ }^{11}$, H. L. Gomez ${ }^{11}$, M. A. T. Groenewegen ${ }^{12}$, R. Indebetouw $^{13,14}$,

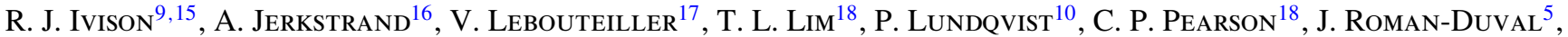 \\ P. Royer ${ }^{19}$, Lister Staveley-Smith ${ }^{20,21}$, B. M. Swinyard ${ }^{1,18}$, P. A. M. van Hoof ${ }^{12}$, J. Th. VAn Loon ${ }^{22}$, Joris VerstapPen ${ }^{4,23}$, \\ Roger Wesson ${ }^{24}$, Giovanna Zanardo ${ }^{20}$, Joris A. D. L. Blommaert ${ }^{19,25}$, Leen Decin $^{19}$, W. T. ReaCH ${ }^{26}$, \\ George Sonneborn ${ }^{2}$, Griet C. Van de Steene ${ }^{12}$, and Jeremy A. Yates ${ }^{1}$ \\ ${ }^{1}$ Department of Physics and Astronomy, University College London, Gower Street, London WC1E 6BT, UK; mikako@ star.ucl.ac.uk \\ ${ }^{2}$ Observational Cosmology Laboratory Code 665, NASA Goddard Space Flight Center, Greenbelt, MD 20771, USA \\ ${ }^{3}$ Department of Astronomy, 475 North Charter Street, University of Wisconsin, Madison, WI 53706, USA \\ ${ }^{4}$ Sterrenkundig Observatorium, Universiteit Gent, Krijgslaan 281 S9, B-9000 Gent, Belgium \\ ${ }^{5}$ Space Telescope Science Institute, 3700 San Martin Drive, Baltimore, MD 21218, USA \\ ${ }^{6}$ Departamento de Astrofísica, Centro de Astrobiología, CSIC-INTA, Ctra. de Torrejón a Ajalvir km 4, E-28850 Madrid, Spain \\ ${ }^{7}$ Department of Physics and Astronomy, Louisiana State University, Baton Rouge, LA 70803, USA \\ ${ }^{8}$ Department of Physics and Astronomy, University of Canterbury, Private Bag 4800, Christchurch 8140, New Zealand \\ ${ }^{9}$ SUPA, Institute for Astronomy, University of Edinburgh, Blackford Hill, Edinburgh EH9 3HJ, UK \\ ${ }^{10}$ The Oskar Klein Centre, Department of Astronomy, Stockholm University, Albanova, SE-10691 Stockholm, Sweden \\ ${ }^{11}$ School of Physics and Astronomy, Cardiff University, Cardiff CF24 3AA, UK \\ ${ }^{12}$ Koninklijke Sterrenwacht van België, Ringlaan 3, 1180 Brussel, Belgium \\ ${ }^{13}$ Department of Astronomy, University of Virginia, P.O. Box 400325, Charlottesville, VA 22904, USA \\ ${ }^{14}$ National Radio Astronomy Observatory, 520 Edgemont Road, Charlottesville, VA 22903, USA \\ ${ }^{15}$ European Southern Observatory, Karl-Schwarzschild-Strasse 2, D-85748 Garching, Germany \\ ${ }^{16}$ Astrophysics Research Centre, School of Mathematics and Physics, Queen's University Belfast, Belfast BT7 1NN, UK \\ 17 AIM, CEA/Saclay, L'Orme des Merisiers, F-91191 Gif-sur-Yvette, France \\ ${ }^{18}$ RAL Space, Rutherford Appleton Laboratory, Chilton, Didcot, Oxfordshire OX11 0QX, UK \\ ${ }^{19}$ Instituut voor Sterrenkunde, Katholieke Universiteit Leuven, Celestijnenlaan 200D, B-3001 Leuven, Belgium \\ ${ }^{20}$ International Centre for Radio Astronomy Research (ICRAR), M468, The University of Western Australia, Crawley, WA 6009, Australia \\ ${ }^{21}$ Australian Research Council, Centre of Excellence for All-sky Astrophysics (CAASTRO) \\ ${ }^{22}$ Lennard Jones Laboratories, Keele University, Keele, Staffordshire ST5 5BG, UK \\ ${ }^{23}$ Kapteyn Astronomical Institute, P.O. Box 800, 9700 AV Groningen, The Netherlands \\ ${ }^{24}$ European Southern Observatory, Alonso de Córdova 3107, 19001 Casilla, Santiago, Chile \\ ${ }^{25}$ Astronomy and Astrophysics Research Group, Department of Physics and Astrophysics, \\ Vrije Universiteit Brussel, Pleinlaan 2, B-1050 Brussels, Belgium \\ ${ }^{26}$ Stratospheric Observatory for Infrared Astronomy, Universities Space Research Association, MS 232-12, \\ NASA/Ames Research Center, Moffett Field, CA 94035, USA \\ Received 2014 August 22; accepted 2014 November 18; published 2015 February 9
}

\begin{abstract}
We present new Herschel photometric and spectroscopic observations of Supernova 1987A, carried out in 2012. Our dedicated photometric measurements provide new $70 \mu \mathrm{m}$ data and improved imaging quality at 100 and $160 \mu \mathrm{m}$ compared to previous observations in 2010. Our Herschel spectra show only weak CO line emission, and provide an upper limit for the $63 \mu \mathrm{m}[\mathrm{O} \mathrm{I}]$ line flux, eliminating the possibility that line contaminations distort the previously estimated dust mass. The far-infrared spectral energy distribution (SED) is well fitted by thermal emission from cold dust. The newly measured $70 \mu \mathrm{m}$ flux constrains the dust temperature, limiting it to nearly a single temperature. The far-infrared emission can be fitted by $0.5 \pm 0.1 M_{\odot}$ of amorphous carbon, about a factor of two larger than the current nucleosynthetic mass prediction for carbon. The observation of $\mathrm{SiO}$ molecules at early and late phases suggests that silicates may also have formed and we could fit the SED with a combination of $0.3 M_{\odot}$ of amorphous carbon and $0.5 M_{\odot}$ of silicates, totalling $0.8 M_{\odot}$ of dust. Our analysis thus supports the presence of a large dust reservoir in the ejecta of SN 1987A. The inferred dust mass suggests that supernovae can be an important source of dust in the interstellar medium, from local to high-redshift galaxies.
\end{abstract}

Key words: dust, extinction - infrared: ISM - infrared: stars - ISM: supernova remnants - submillimeter: ISM submillimeter: stars - supernovae: individual (Supernova 1987A)

\footnotetext{
* Herschel is an ESA space observatory with science instruments provided by European-led Principal Investigator consortia and with important participation from NASA. PACS has been developed by a consortium of institutes led by MPE (Germany) and including UVIE (Austria); KU Leuven, CSL, IMEC (Belgium); CEA, LAM (France); MPIA (Germany); INAF-IFSI/OAA/OAP/ OAT, LENS, SISSA (Italy); IAC (Spain). This development has been supported by the funding agencies BMVIT (Austria), ESA-PRODEX (Belgium), CEA/CNES (France), DLR (Germany), ASI/INAF (Italy), and CICYT/MCYT (Spain). SPIRE has been developed by a consortium of institutes led by Cardiff University (UK) and including Univ. Lethbridge (Canada); NAOC (China); CEA, LAM (France); IFSI, Univ. Padua (Italy); IAC (Spain); Stockholm Observatory (Sweden); Imperial College London,
}

\section{INTRODUCTION}

The explosion of supernova (SN) 1987A in the Large Magellanic Cloud (LMC) was detected on 1987 February 23 (Kunkel et al. 1987). SN 1987A has since provided a unique opportunity to study the evolution of SN ejecta and SN remnants.

RAL, UCL-MSSL, UKATC, Univ. Sussex (UK); and Caltech, JPL, NHSC, Univ. Colorado (USA). This development has been supported by national funding agencies: CSA (Canada); NAOC (China); CEA, CNES, CNRS (France); ASI (Italy); MCINN (Spain); SNSB (Sweden); STFC and UKSA (UK); and NASA (USA). 
One of the early discoveries was the detection of thermal emission from dust, believed to have formed in the ejecta. The emission, appearing at mid-infrared wavelengths, probably began at about day 260, increasing in flux to day 1316 (Wooden et al. 1993; Bouchet et al. 1991). The emission was attributed to $\sim 10^{-4} M_{\odot}$ of dust. About 23 yr later, the Herschel Space Observatory detected thermal dust emission at $100-350 \mu \mathrm{m}$ (Matsuura et al. 2011), with ALMA resolved images confirming that the far-infrared emitting dust was located in the ejecta (Indebetouw et al. 2014). The Herschel-based dust mass was three orders of magnitude larger $\left(0.4-0.7 M_{\odot}\right)$ than previously reported. This surprisingly large dust mass triggered debates about the nature of the far-infrared emission, not only because it was far larger than the measurements made at much earlier epochs, but also because it was much larger than typical dust masses that had been deduced from Spitzer mid-infrared observations of other core-collapse $\mathrm{SNe}$ during their first three years after outburst, $10^{-6}$ to $10^{-4} M_{\odot}$ (e.g., Gall et al. 2011), although Herschel far-infrared observations of two historical SN remnants, Cassiopeia A and the Crab Nebula (e.g., Barlow et al. 2010; Gomez et al. 2012) found significantly larger dust masses $\left(\geqslant 0.1 M_{\odot}\right)$. Possible ways to reduce the dust mass derived for SN 1987A have been proposed, including line contamination in the Herschel filter band-passes. Because the initial detection was made from fast-scan observations (leading to spatial undersampling for PACS) during the Herschel HERITAGE survey of the LMC (Meixner et al. 2013), and because possible line contamination needed to be evaluated, we obtained Herschel dedicated PACS and SPIRE observations of SN 1987A in 2012 using both their photometric and spectroscopic modes, which we report here.

\section{OBSERVATIONS AND DATA REDUCTION}

\subsection{PACS and SPIRE Imaging}

The Herschel Space Observatory (Pilbratt et al. 2010) detected SN 1987A at far-infrared and submillimeter wavelengths in 2010 (Matsuura et al. 2011), as part of the HERschel Inventory of the Agents of Galaxy Evolution (HERITAGE; Meixner et al. 2013). The survey used five filter bands from 100 to $500 \mu \mathrm{m}$ and SN 1987A was detected in the four bands from 100 to $350 \mu \mathrm{m}$. Herschel scanned SN 1987A on 2010 April 30 and 2010 August 5 (days 8467 and 8564 after the explosion). We adopt the SPIRE 250 and $350 \mu \mathrm{m}$ fluxes from the HERITAGE point source cata$\log$ (Meixner et al. 2013). The HERITAGE PACS images were affected by residual striping due to $1 / f$ noise (Meixner et al. 2013) and dedicated stripe removal procedures were adopted for the image reconstruction for SN 1987A.

Dedicated Herschel photometric observations were carried out in 2012, acquired as part of a guaranteed time observing programme (GT2_mbaes_3). The PACS (Poglitsch et al. 2010) images (ObsID 1342237428, 1342237429, 1342237430, and 1342237431) were acquired on 2012 January 13 (UT), corresponding to day 9090 after the explosion. The large scan map mode was used. The observing sequence was composed of two observations, one to obtain 100 and $160 \mu \mathrm{m}$ cross-scan images with a $2 \times 1295 \mathrm{~s}$ duration, and the other to obtain 70 and $160 \mu \mathrm{m}$ cross-scan images with a $2 \times 2245$ s duration. The FWHMs of the point spread functions (PSFs) were 5.46 × 5.76, 6.69 × 6.89, and $10.65 \times 12.13 \operatorname{arcsec}^{2}$ for the PACS 70, PACS 100 and PACS 160 bands, respectively (PACS observer's manual ${ }^{27}$ ).

\footnotetext{
27 http://herschel.esac.esa.int/Docs/PACS/html/pacs_om.html
}

Table 1

The Measured Far-infrared and Submillimeter Flux Densities of SN 1987A

\begin{tabular}{lccc}
\hline \hline Band Name & \multicolumn{2}{c}{ Flux (mJy) } & Reference \\
\cline { 2 - 3 } & $\begin{array}{c}\text { HERITAGE } \\
\text { Follow-up }\end{array}$ & \\
\hline PACS $70 \mu \mathrm{m}$ & $\ldots$ & 2012 & \\
PACS $100 \mu \mathrm{m}$ & $98.3 \pm 8.5$ & $82.4 \pm 4.5$ & \\
PACS $160 \mu \mathrm{m}$ & $169.6 \pm 11.1$ & $153.0 \pm 9.0$ & \\
SPIRE $250 \mu \mathrm{m}$ & $123.3 \pm 13.4$ & $110.7 \pm 25.2$ & \\
SPIRE $350 \mu \mathrm{m}$ & $53.8 \pm 18.1$ & $69.3 \pm 22.8$ & \\
SPIRE $500 \mu \mathrm{m}$ & $<57.3(3 \sigma)$ & $<60(3 \sigma)$ & \\
\hline APEX $350 \mu \mathrm{m}$ & $58 \pm 13$ & & Lakićević et al. (2012) \\
ALMA $450 \mu \mathrm{m}$ & & $45 \pm 15$ & Zanardo et al. (2014) \\
ALMA $870 \mu \mathrm{m}$ & & $4.9 \pm 1.6$ & Zanardo et al. (2014) \\
\hline
\end{tabular}

The absolute flux calibration uncertainties of the PACS photometer are estimated to be $3 \%$ for the 70 and $100 \mu \mathrm{m}$ bands, and $5 \%$ for the $160 \mu \mathrm{m}$ band.

The SPIRE (Griffin et al. 2010) images of SN 1987A (OBSID 1342239283) were obtained using the large scan map mode on 2012 February 14 (UT), corresponding to an epoch of 9122 days. With an integration time of $2553 \mathrm{~s}$, we simultaneously obtained $10 \operatorname{arcmin} \times 10 \operatorname{arcmin}$ images at 250,350, and $500 \mu \mathrm{m}$. The FWHMs of the beams were 18.2, 24.9, and 36.3 arcsec at 250, 350 , and $500 \mu \mathrm{m}$, respectively (Griffin et al. 2013) and 11 arcsec pixel scales at 250,350 , and $500 \mu \mathrm{m}$, respectively. The absolute flux calibration errors were estimated to be $5 \%$ (Bendo et al. 2013). The color correction factors are less than $1 \%$, so we ignore them.

Figure 1 shows the PACS and SPIRE images of SN 1987A and its surroundings from the Herschel 2012 observations. SN 1987A was detected as a point source from $70-350 \mu \mathrm{m}$, whereas it was not clearly detected in the SPIRE 500 image, because it is diluted by LMC interstellar medium (ISM) emission in the large $500 \mu \mathrm{m}$ beam.

The IDL PSF-fitting code, starfinder (Diolaiti et al. 2000) was used to obtain point source photometric measurements; details are given by Meixner et al. (2013). The uncertainties in the fluxes include the error maps from the pipeline, the uncertainties in the absolute flux calibration, the fluctuations in the sky level, as well as starfinder's fitting uncertainties.

Table 1 lists the measured Herschel fluxes, which are consistent between 2010 and 2012 within the uncertainties. The pointed observations have reduced uncertainties for the PACS 100 and $160 \mu \mathrm{m}$ fluxes, because the optimized imaging for a point source provides higher sampling rates by a factor of three, improving the overall image quality in the PACS bands. The SPIRE 250 and $350 \mu \mathrm{m}$ fluxes are consistent between the HERITAGE and the pointed observations within the $1 \sigma$ uncertainties, as the uncertainties are dominated by uncertainties of the sky estimates.

Figure 2 presents the spectral energy distribution (SED) of SN 1987A from infrared to millimetre wavelengths. In addition to our Herschel measurements, we assembled additional flux measurements from the literature. The day 9090 mid-infrared photometric points were extrapolated from measurements made on day 7983, adopting the empirical power-law fit of Dwek et al. (2010) to the flux increase with time. The estimated day 9090 fluxes are $15.4 \pm 0.4 \mathrm{mJy}$ and $97 \pm 5 \mathrm{mJy}$ at 8.0 and $24 \mu \mathrm{m}$, respectively. The mid-infrared fluxes have been shown to be due to thermal emission from silicate dust grains located in the equatorial ring (Bouchet et al. 2006). We fitted these fluxes 


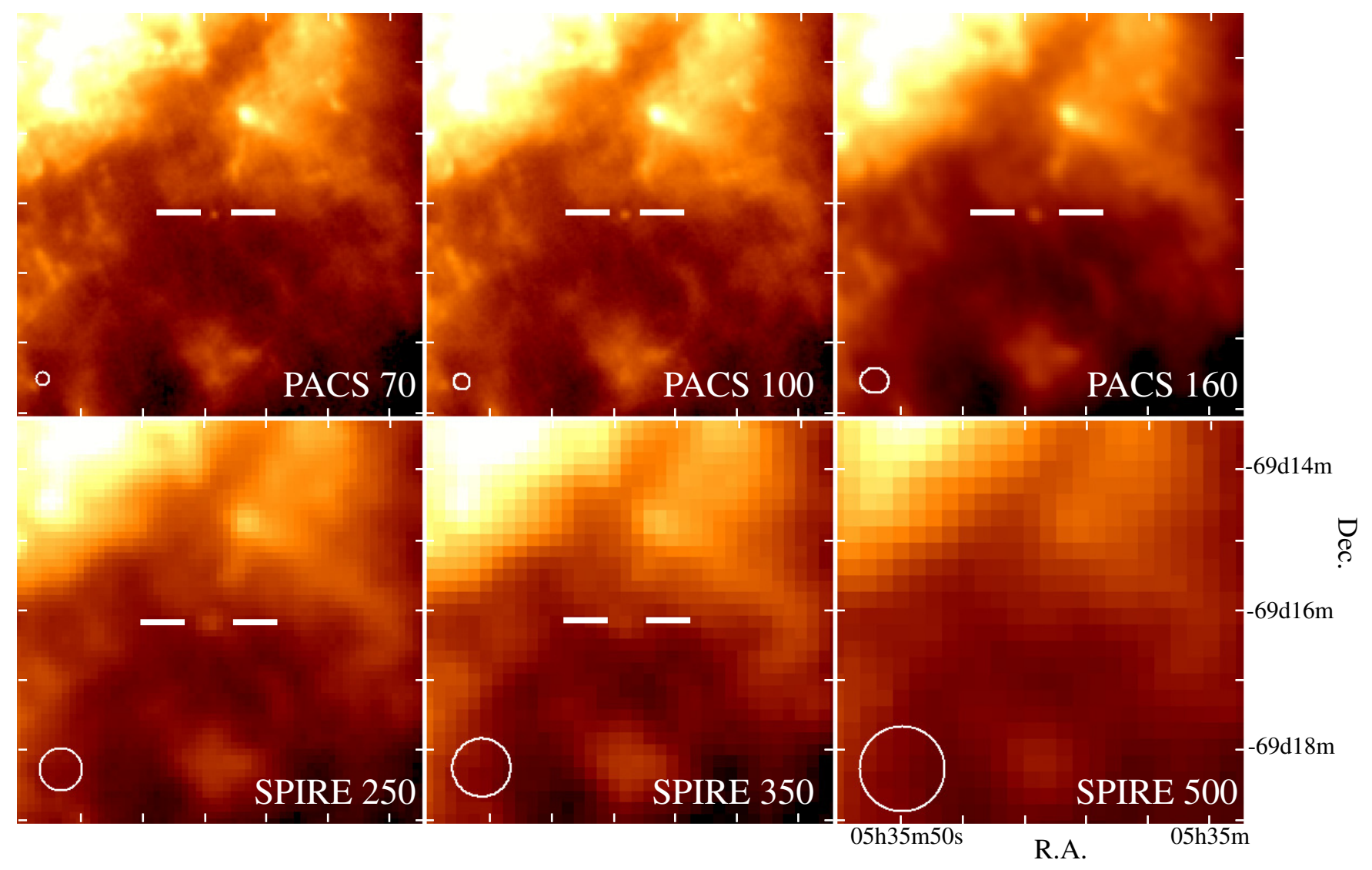

Figure 1. PACS and SPIRE images of SN 1987A and its surroundings from the dedicated observations taken in 2012. The supernova is found as a point source. The white circles show the size of the PSFs.

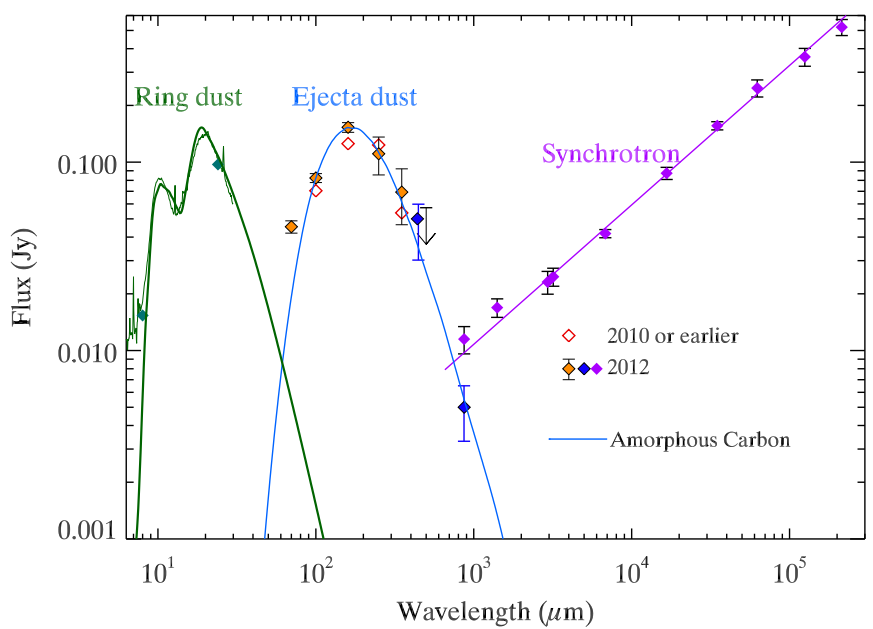

Figure 2. 2010 and 2012 SED of SN1987A, showing two distinct components of thermal dust emission: warm dust from the equatorial ring and cold dust from the ejecta. Additionally, synchrotron radiation from the ring is detected at millimeter wavelengths and longer, showing a power-law frequency dependence (StaveleySmith et al. 2014; Zanardo et al. 2014). Two dust models are plotted-the parameters for the cold ejecta dust can be found in Table 2 (model b), while the silicate fit to the ring dust is plotted as a thick green line (see the text).

with silicate dust emission (Weingartner \& Draine 2001); its day 9090 parameters are a dust temperature of $187 \mathrm{~K}$ and a dust mass of $1.6 \times 10^{-5} M_{\odot}$. The Spitzer IRS spectrum at day 7965 (Dwek et al. 2010), which was estimated to correspond to a dust temperature of $T \sim 175 \mathrm{~K}$, was scaled by $B_{v}(T=187 \mathrm{~K}) / B_{v}(T=$ $175 \mathrm{~K})$, where $B_{v}$ is the Planck function, and is plotted as the thin green line in Figure 2. The millimetre-wavelength fluxes are fitted by a synchrotron radiation spectrum; these fluxes increase in time and the fluxes were scaled to day 9090 from recent ATCA observations (Zanardo et al. 2014), or scaled to the Herschel epoch via exponential fitting of the flux densities from day 8000 (Zanardo et al. 2010; Staveley-Smith et al. 2014; G. Zanardo et al., in preparation).

\subsection{The SPIRE Fourier Transform Spectrum}

A 447-1540 GHz SPIRE Fourier Transform spectrum of SN 1987A was obtained on 2012 June 12 (day 9241; ObsID 1342246989) in a Herschel guaranteed time program (GT2_mbarlow_1) with a total duration of $14156 \mathrm{se}$, and a spectral resolution of $1.2 \mathrm{GHz}$. The spectrum was reduced in HIPE v11, and part of the spectrum was presented by Kamenetzky et al. (2013). For the $J=6-5$ and 7-6 $\mathrm{CO}$ lines the measured line fluxes were $(7 \pm 3) \times 10^{-18}$ and $(8 \pm 2) \times 10^{-18} \mathrm{~W} \mathrm{~m}^{-2}$. Together with ALMA data, Kamenetzky et al. (2013) estimated a CO temperature between $13 \mathrm{~K}$ and $132 \mathrm{~K}$ and a minimum $\mathrm{CO}$ mass of $0.01 M_{\odot}$.

We calculated the maximum possible $\mathrm{CO}$ contribution to the Herschel photometric bands, adopting an excitation temperature of $132 \mathrm{~K}$, using an LTE code (Matsuura et al. 2002). An upper limit of $<9 \%(3 \sigma)$ is estimated for the CO line contribution to the in-band SPIRE $350 \mu \mathrm{m}$ flux, with other bands having $<3 \%$. Hence we ignore contamination by $\mathrm{CO}$ lines of the broad-band photometric points.

\subsection{PACS Observations of the [O I] $63 \mu \mathrm{m}$ Line Region}

Targeting the [O I] $63 \mu \mathrm{m}$ line, a PACS 59-70 $\mu \mathrm{m}$ spectrum of SN 1987A was obtained on 2012 October 23 (day 9374; 


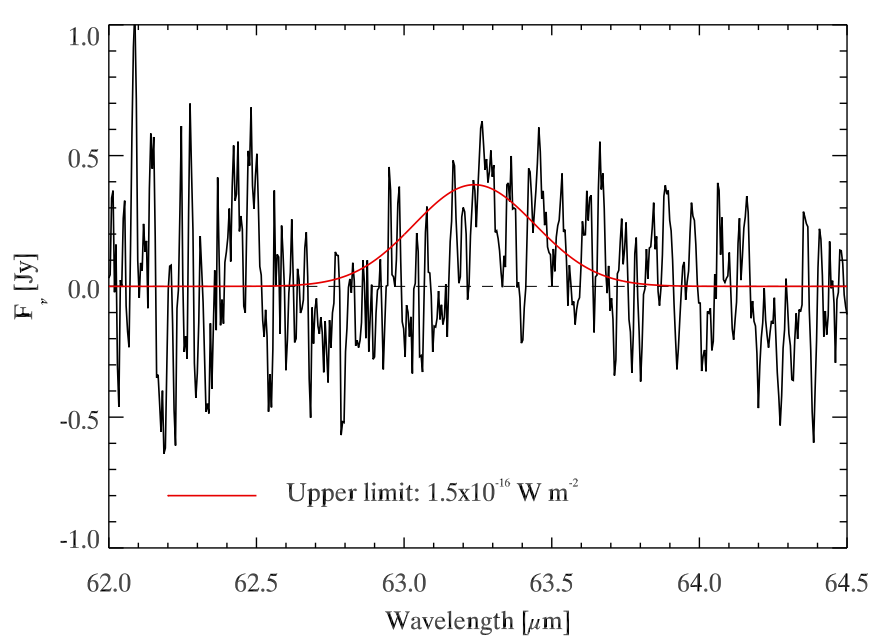

Figure 3. 2012 PACS spectrum of SN 1987A showing the region of the $63 \mu \mathrm{m}$ [O I ] line, which is not detected. The continuous line corresponds to our $3 \sigma$ line flux upper limit.

ObsID 1342237430), as part of the MESS guaranteed time key program (Groenewegen et al. 2011), using chopped-nodded PACS range spectroscopy modes (Poglitsch et al. 2010). The spectral resolution was $80 \mathrm{~km} \mathrm{~s}^{-1}(R=1500)$. The total duration was $3143 \mathrm{~s}$ for four scan-repetitions.

Figure 3 shows the PACS spectrum and the non-detection of the [O I] $63 \mu \mathrm{m}$ line. An upper limit of $<1.5 \times 10^{-16} \mathrm{~W} \mathrm{~m}^{-2}$ is obtained for the case of a $2300 \mathrm{~km} \mathrm{~s}^{-1}$ FWHM ejecta line width (Kjær et al. 2010). The upper limit for the [O I] line flux is consistent with model predictions, where the models of Kozma \& Fransson (1998) and Groningsson et al. (2008) predict [O I] line intensities of $(0.5-1) \times 10^{-16} \mathrm{~W} \mathrm{~m}^{-2}$, while Jerkstrand et al. (2011) predict $1.3 \times 10^{-16} \mathrm{~W} \mathrm{~m}^{-2}$ from the ejecta in 2012 .

For line emission originating from the ring, an upper limit of $<2 \times 10^{-17} \mathrm{~W} \mathrm{~m}^{-2}$ was obtained, assuming a line width of $350 \mathrm{~km} \mathrm{~s}^{-1}$ (e.g., Groningsson et al. 2008). This is within the range expected $\left(<5 \times 10^{-18} \mathrm{~W} \mathrm{~m}^{-2}\right)$ from adopting the predicted $63 \mu \mathrm{m} / 6300 \AA$ line ratio of Groningsson et al. (2008), and the observed [O I] $6300 \AA$ flux decrease with time (Migotto et al., in preparation).

\section{ANALYSIS}

Figure 2 shows the SED of SN 1987A. The newly obtained photometric point at $70 \mu \mathrm{m}$ clearly shows the dip between two discrete components of thermal dust emission (warm and cold). These components arise from two different locations within the system: the cold dust is located in the ejecta (Indebetouw et al. 2014), while the warm dust is emitted in/near the equatorial ring associated with circumstellar material from the progenitor star (Bouchet et al. 2006).

We fitted the cold component of the thermal dust emission in order to estimate the required temperatures and dust masses for different grain compositions. The fluxes from $100-870 \mu \mathrm{m}$ in 2012 can be fitted with dust having a single temperature and a single composition (Figure 2). The fitting was optimized using the $\chi^{2}$ minimum fitting code MPFIT in IDL.

Dust optical constants were taken from Zubko et al. (1996) for amorphous carbon (ACAR), from Jäger et al. (2003) for silicate, and from Begemann et al. (1994, 1997) for aluminum oxide $\left(\mathrm{Al}_{2} \mathrm{O}_{3}\right)$ and sulfides $\left(\mathrm{Mg}_{0.9} \mathrm{Fe}_{0.1} \mathrm{~S}\right.$ and $\left.\mathrm{FeS}\right)$. Unfortunately, the wavelength coverage of the $\mathrm{Al}_{2} \mathrm{O}_{3}$ data is too short $(<200 \mu \mathrm{m})$ and the $\mathrm{Mg}_{0.9} \mathrm{Fe}_{0.1} \mathrm{~S}$ data are noisy beyond $150 \mu \mathrm{m}$, so that these analyses are for general guidance only. Grain densities $(\rho)$ of $1.81,3.3,3.2$ and $4.83 \mathrm{~g} \mathrm{~cm}^{-3}$ were adopted for amorphous carbon, silicates, $\mathrm{Al}_{2} \mathrm{O}_{3}$ and $\mathrm{FeS}$, respectively (Zubko et al. 2004; Draine \& Lee 1984; Begemann et al. 1997; Semenov et al. 2003). $\mathrm{Mg}_{0.9} \mathrm{Fe}_{0.1} \mathrm{~S}$ is a similar material to $\mathrm{MgS}$, with a grain density of $2.84 \mathrm{~g} \mathrm{~cm}^{-3}$ (Gail \& Sedlmayr 2014). We calculated the dust emissivity $\left(Q_{v}\right)$ at frequency $v$ using Mie theory, and with dust mass absorption coefficients $\left(\kappa_{v}=3 Q_{v} / 4 \rho a_{d}\right)$. A grain size, $a_{d}$ of $0.1 \mu \mathrm{m}$ is assumed. However, $\kappa_{v}$ and thus the inferred dust mass, does not depend on $a$ up to $a=0.5 \mu \mathrm{m}$. The only exception is iron, for which we have used $a$-dependent iron emissivities (Nozawa et al. 2006).

Our thermal dust emission calculations consider both optically thin and thick cases. In the optically thin case, the flux density $F_{v}$ from a dust mass $\left(M_{d}\right)$ is given by $F_{v}=$ $M_{d}\left(4 \kappa_{\nu} \pi B_{v} / 4 \pi d^{2}\right)$ (Hildebrand 1983), where $d$ is the distance to the LMC, adopted to be $50 \mathrm{kpc}$. In order to deal with the optically thick cases, we use the escape probability $\left(P_{\nu}\right)$ for a photon emitted in a sphere (Osterbrock 1989). The optical depth $\left(\tau_{v}\right)$ for a sphere was calculated using the equation $\tau_{0}(\nu)=3 / 4\left(M_{d} / \pi R^{2}\right) \kappa_{\nu}$, where $\tau_{0}(\nu)$ is the radial optical depth along the line of sight, and a radius $(R)$ of $1 \times 10^{17} \mathrm{~cm}$ was assumed (Indebetouw et al. 2014). The flux density is given by $F_{v}^{\prime}=F_{v} P_{v}$.

Our dust model fitting results are shown in Figure 4 and summarized in Table 2. Models (a)-(j) omitted the $70 \mu \mathrm{m}$ flux point from the fits, while models $(\mathrm{k})-(\mathrm{n})$ included it. For amorphous carbon the single temperature component fitting yielded a dust mass of $0.5 \pm 0.1 M_{\odot}$, with little difference in the inferred dust masses between the optically thick and optically thin cases (models a and b). This is because the emission is largely optically thin, being marginally optically thick at $100 \mu \mathrm{m}$ $\left(\tau_{100 \mu \mathrm{m}}=1.2\right)$. Fitting with silicates requires the use of escape probabilities, as $\tau>1$ at wavelengths $\leqslant 160 \mu \mathrm{m}$.

Overall, our fitted dust models produce dust masses and temperatures consistent with the previous Herschel analysis for amorphous carbon and iron (Matsuura et al. 2011). A slight difference is found in the dust temperature inferred for silicates, partly because the higher signal-to-noise 2012 PACS photometer fluxes are slightly different from those measured in 2010, partly because different dust optical constants are used, and partly because previously an optically thin case was assumed, which is now found not to be valid at 100 and $160 \mu \mathrm{m}$.

The $\chi^{2}$ values are the lowest for amorphous carbon, suggesting that this dust species could be the major component of the ejecta dust. Silicates, whose emissivities decline more steeply toward longer wavelengths than amorphous carbon, show larger $\chi^{2}$ values than amorphous carbon.

We could also fit the SED using a combination of silicates and amorphous carbon (Figure 4(d)); the combination required $0.5 M_{\odot}$ of silicate and $0.3 M_{\odot}$ of amorphous carbon, for a combined dust mass of $0.8 M_{\odot}$ (Table 2).

The fits discussed so far did not include the $70 \mu \mathrm{m}$ flux measured in 2012. This flux has potential contaminants. Our Herschel PACS spectrum shows that the [O I] $63 \mu \mathrm{m}$ line could contribute up to $24 \%$ of the $70 \mu \mathrm{m}$ in-band flux (Section 2.3). Further, the contribution from "warm" dust located in the equatorial ring was estimated to be about $\sim 12 \%$ of the in-band flux (Section 2.1), corresponding to a combined contribution of up to $36 \%$ of the $70 \mu \mathrm{m}$ in-band flux, unrelated to the thermal emission of cold dust. We fitted the far-infrared SED with cold dust emission, including the $70 \mu \mathrm{m}$ point in the fit, after subtracting off $36 \%$ of the $70 \mu \mathrm{m}$ in-band flux. First, we fitted with a single dust component. The fit used amorphous carbon 


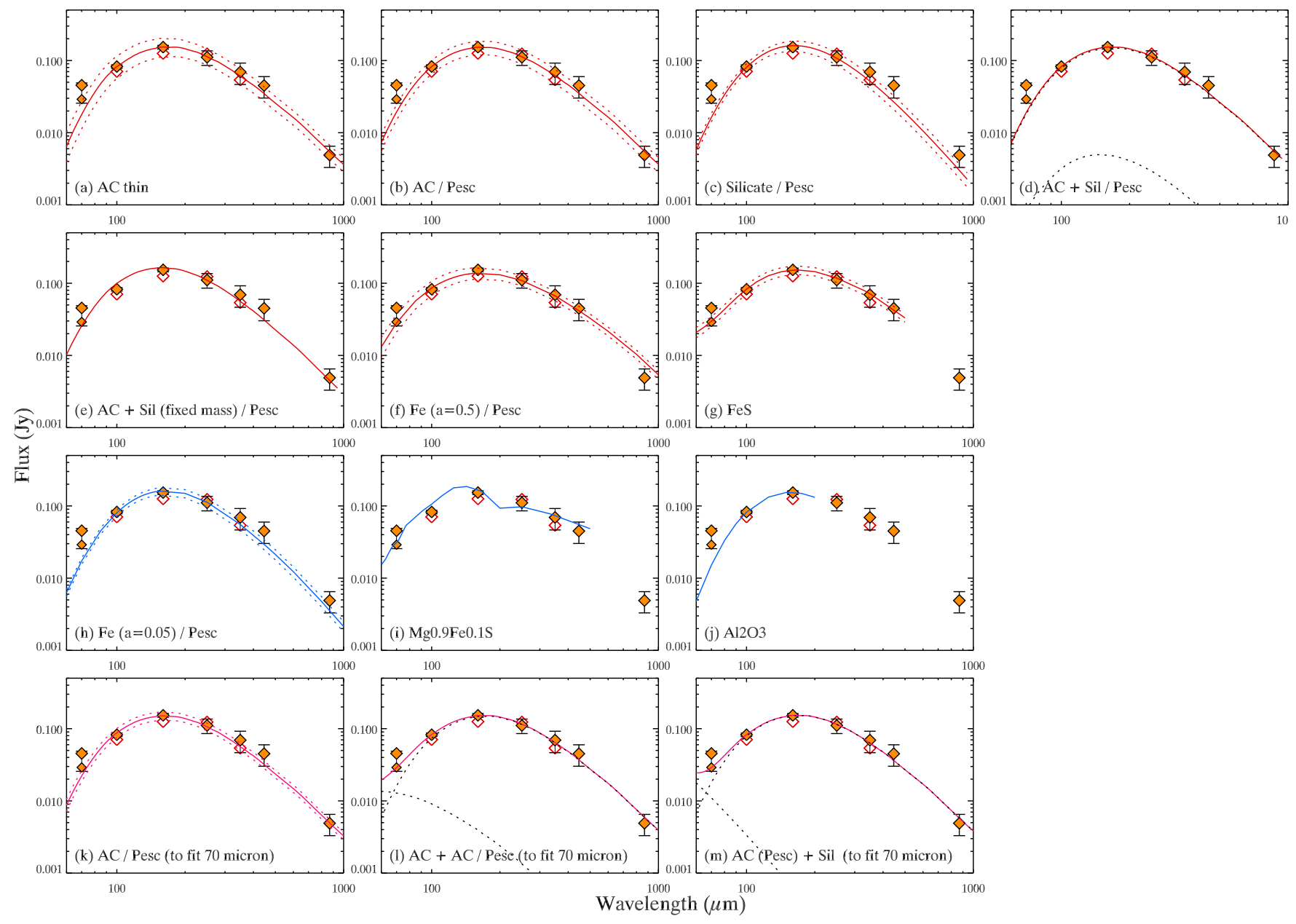

Figure 4. Model SED fits. Diamonds show the observed fluxes in 2012, as in Figure 2. The lower error bar limit and smaller diamonds of the $70 \mu \mathrm{m}$ flux includes estimated contribution from [O I] and ring dust; the $70 \mu \mathrm{m}$ flux with these contributions subtracted is indicated by filled orange diamonds. Open diamonds show the measured fluxes in 2010. The fits are plotted as solid curves, and their uncertainty ranges are shown as dotted curves. The fit for $\mathrm{Al}_{2} \mathrm{O}_{3}$ in box (i) stops at $200 \mu \mathrm{m}$, the longest wavelength for which optical constants are available (see the text). The parameters for each of the fits can be found in Table 2 . The label $P_{\text {esc }}$ indicates that the fitting involves escape probabilities. The models $(\mathrm{h}-\mathrm{j})$, plotted in blue lines, have fitted dust masses that are a factor of five larger than dust mass constraints predicted by nuclear synthesis models.

Table 2

Summary of the Derived Dust Masses and Dust Mass Constraints from Predicted Available Elemental Masses

\begin{tabular}{|c|c|c|c|c|c|c|}
\hline & Model & $P_{v}$ & $\begin{array}{c}M_{d} \\
\left(M_{\odot}\right)\end{array}$ & $\begin{array}{c}T_{d} \\
(\mathrm{~K})\end{array}$ & Reduced $\chi^{2}$ & $\begin{array}{c}M_{m} \\
\left(M_{\odot}\right)\end{array}$ \\
\hline (a) & Amorphous carbon (AC) & $\cdots$ & $0.5 \pm 0.1$ & $20.3 \pm 0.5$ & 0.14 & 0.25 \\
\hline (b) & $\mathrm{AC}$ & $P_{v}$ & $0.5 \pm 0.1$ & $23.2 \pm 0.5$ & 0.13 & 0.25 \\
\hline (c) & Silicate (sil) & $P_{v}$ & $2.4 \pm 0.5$ & $22.5 \pm 0.3$ & 0.77 & $\sim 0.5$ \\
\hline (d) & $\mathrm{AC}+$ silicate & $P_{v}$ & $0.5(\mathrm{AC})+0.07(\mathrm{sil})$ & $23(\mathrm{AC})+22(\mathrm{sil})$ & 0.13 & \\
\hline (e) & $\mathrm{AC}+$ silicate & $P_{v}$ & $0.3(\mathrm{AC})+0.5$ (sil) fixed & $25(\mathrm{AC})+20($ sil $)$ & 1.15 & \\
\hline (f) & $\mathrm{Fe}\left(a_{d}=0.5 \mu \mathrm{m}\right)$ & $P_{v}$ & $0.37 \pm 0.04$ & $26.9 \pm 0.7$ & 1.57 & 0.24 \\
\hline (g) & $\mathrm{FeS}$ & $\cdots$ & $0.9 \pm 0.1$ & $31 \pm 0.2$ & 0.86 & 0.24 \\
\hline \multicolumn{7}{|c|}{ Less important for far-infrared emission } \\
\hline (h) & $\mathrm{Fe}\left(a_{d}=0.05 \mu \mathrm{m}\right)$ & $P_{v}$ & $12.8 \pm 2.0$ & $22.8 \pm 0.2$ & 0.44 & 0.24 \\
\hline (i) & $\mathrm{Mg}_{0.9} \mathrm{Fe}_{0.1} \mathrm{~S}$ & $\cdots$ & 1.4 & 19.5 & 6.33 & 0.25 \\
\hline (j) & $\mathrm{Al}_{2} \mathrm{O}_{3}$ & $\cdots$ & 0.7 & 20 & & 0.02 \\
\hline \multicolumn{7}{|c|}{ Testing fits to $70-870 \mu \mathrm{m}$ flux, after $36 \%$ of the $70 \mu \mathrm{m}$ flux was subtracted } \\
\hline$(\mathrm{k})$ & $\mathrm{AC}$ & $P_{v}$ & $0.4 \pm 0.04$ & $23.7 \pm 0.4$ & 0.96 & \\
\hline (1) & $\mathrm{AC}+\mathrm{AC}$ & $P_{v}$ & $0.5($ cold $)+5 \times 10^{-4}($ warm $)$ & $24+83$ & 0.12 & \\
\hline (m) & $\mathrm{AC}+$ Silicate & $P_{v}$ (AC only) & $0.5(\mathrm{AC})+6 \times 10^{-5}(\mathrm{sil})$ & $23(\mathrm{AC})+134($ sil $)$ & 0.11 & \\
\hline
\end{tabular}

Notes. $P_{v}$ shows whether escape probabilities were involved in the calculation. $M_{d}$ : dust mass, $T_{d}$ : dust temperature, and $a_{d}$ : grain radius, which is $0.1 \mu \mathrm{m}$, unless stated. $M_{m}$ : maximum dust mass allowed by elemental masses predicted by current explosive nucleosynthesis models (Rauscher et al. 2002). Fe elemental mass includes decay from ${ }^{56} \mathrm{Ni}$ to ${ }^{56} \mathrm{Co}$, then to ${ }^{56} \mathrm{Fe}$ with half lives of 6 and 77 days. Model (e) used fixed dust masses. 
and the optically thick assumption was applied. The result is shown in Figure 4(k), with a dust temperature of $23.7 \pm 0.4 \mathrm{~K}$ and a dust mass of $0.4 \pm 0.04 M_{\odot}$. This dust mass and its temperature are nearly consistent within the $1 \sigma$ uncertainties with the fit that omitted the $70-\mu \mathrm{m}$ flux point (Table 2), but the fit is poor at $70 \mu \mathrm{m}$ and noticeably worse at 250 and $350 \mu \mathrm{m}$, compared to the single component fit that omitted the $70 \mu \mathrm{m}$ point (Figure 4(b)).

We therefore also produced fits to the entire $70-870 \mu \mathrm{m}$ SED using two dust components. The results are shown in Figures $4(\mathrm{k})-(\mathrm{m})$ and the parameters are listed in Table 2. The fitting procedure found a solution with a warm silicate component having a temperature of $134 \mathrm{~K}$ and a dust mass of $6 \times 10^{-5} M_{\odot}$, along with a cold amorphous carbon component having a temperature of $24 \mathrm{~K}$ and a dust mass of $0.5 \mathrm{M}_{\odot}$. Since the temperature of the warm component is close to that discussed earlier for the equatorial ring dust, this suggests that $36 \%$ represents an underestimate of the combined contribution to the $70 \mu \mathrm{m}$ in-band flux made by the $63 \mu \mathrm{m}$ line and by the warm ring dust. The cold AC dust component in the 70-870 $\mu \mathrm{m}$ one and two-component fit has a consistent dust mass for amorphous carbon for the single-component $100-870 \mu \mathrm{m}$ fit.

A possible way to reduce the required mass of silicate dust is to assume that the dust grains are elongated, which can enhance their far-infrared emissivity. For ellipsoid grains with three axes $(a, b, c)$ with $a=b \ll c \ll \lambda$, where $\lambda$ is the emitting wavelength (Hoyle \& Wickramasinghe 1991), we find that for silicates the value of $\kappa$ is enhanced by a constant factor of about two for wavelengths longer than $\sim 50 \mu \mathrm{m}$. The value of $\kappa$ for elongated amorphous carbon grains is enhanced by a factor of $\sim 20-200$ from $\sim 50$ to $1000 \mu \mathrm{m}$. However, the resulting wavelength dependence of $\kappa$ is considerably flattened, providing a very poor fit to the Herschel and ALMA data. For silicates, the dust mass in SN1987A can therefore be $0.8 M_{\odot}$, if the nucleating silicates attain an elongated shape, but the emission is unlikely to be due to elongated amorphous carbon grains. Grains nucleating in a radioactive environment may acquire an electric charge that can affect the formation and growth of these dust grains. This effect may preferentially affect the silicate grains: because of their dielectric nature, which may cause them to attain an asymmetrical charge distribution; and because they nucleate in an environment that is more directly exposed to hard radiation. The presence of non-spherical grains in dense regions of the ISM has been inferred from observations of linearly polarized thermal dust emission at submillimeter wavelengths (Hildebrand \& Dragovan 1995). The possible presence SNcondensed elongated silicates in the diffuse ISM may require modification to existing interstellar dust models (Zubko et al. 2004; Draine \& Li 2007).

\section{DISCUSSION}

Our single component model fits to the far-infrared SED with amorphous carbon give a dust mass of $0.5 \pm 0.1 M_{\odot}$ (Table 2), while the fit with a mixture of amorphous carbon and silicates requires $0.3 M_{\odot}$ of carbon. These masses of amorphous carbon are higher than the mass of carbon $\left(0.25 M_{\odot}\right)$ currently predicted by explosive nucleosynthesis models for a $19 M_{\odot}$ star (Rauscher et al. 2002), apparently implying a deficit of carbon to account for the far-infrared emission, although the carbon deficit is small $\left(0.05 M_{\odot}\right)$ in the case of the mixed AC+silicate model. We note that that the $\mathrm{C} / \mathrm{O}$ ratios predicted by nucleosynthesis models for core-collapse supernovae (CCSNe) may not be accurate for all initial mass cases. For example, the Crab Nebula progenitor star has been estimated to have had an initial main sequence mass of 9-13 $M_{\odot}$ (Hester 2008; Smith 2013). CCSN yield predictions for 11-13 $M_{\odot}$ initial mass models, the lowest masses for which nucleosynthesis predictions are currently available, all predict $\mathrm{C} / \mathrm{O}$ mass ratios of much less than unity (Woosley \& Weaver 1995; Thielemann et al. 1996; Nomoto et al. 2006), as do higher mass models, whereas the photionization modeling analysis by MacAlpine \& Satterfield (2008) of many locations in the Crab Nebula found the nebula to be overwhelmingly carbon-rich $(\mathrm{C} / \mathrm{O}>1$, both by number and by mass).

Although a $10 \mu \mathrm{m}$ or $18 \mu \mathrm{m}$ silicate emission or absorption feature was not seen during the first three years of SN 1987A's evolution, when its dust SED peaked at mid-infrared wavelengths (Wooden et al. 1993), indications that silicate dust may have formed as well as carbon dust comes from the fact that molecular $\mathrm{SiO}$ vibrational emission was detected at early times (since day 164; Roche et al. 1991), but disappeared from mid-infrared spectra at about the time that dust formation began (Wooden et al. 1993), potentially due to depletion caused by silicate dust formation (Sarangi \& Cherchneff 2013). Recent ALMA submillimeter observations of SN 1987A have also detected $\mathrm{SiO}$, via its rotational emission spectrum (Kamenetzky et al. 2013). The current SED can be fitted with $2.9 \pm 0.5 M_{\odot}$ of silicate grains alone (Figure 4(c)), but this exceeds by a factor of six the silicate mass limit $\left(\sim 0.5 M_{\odot}\right)$ from the $\mathrm{Si}$, $\mathrm{Fe}$ and $\mathrm{Mg}$ abundances predicted by explosive nucleosynthesis models (Thielemann et al. 1990; Rauscher et al. 2002). Hence, it seems implausible for only silicate dust to be present in the ejecta. We have shown however that a combination of $0.3 M_{\odot}$ of amorphous carbon and $0.5 M_{\odot}$ of silicates, for a total dust mass of $0.8 M_{\odot}$, can fit the observed SED while satisfying the currently predicted elemental abundance limits for silicates and is close to the currently predicted elemental mass limit for carbon. If one disregards the predicted elemental carbon mass limit of $0.25 M_{\odot}$, then the lowest total dust mass that can fit the current SED of SN 1987A is $0.5 \pm 0.1 M_{\odot}$ of amorphous carbon.

The value of $\kappa$ for iron grains has a steep dependence on grain radius $a_{d}$. For $a_{d}=0.5 \mu \mathrm{m}$, the derived dust mass is $0.3 M_{\odot}$, but it increases to $14 M_{\odot}$ for $a_{d}=0.05 \mu \mathrm{m}$ (Table 2). As it is unlikely for all iron grains to have a radius of $0.5 \mu \mathrm{m}$, we consider that iron grains are not the major source of the far-infrared emission.

Although Sarangi \& Cherchneff (2013) predicted $\mathrm{Al}_{2} \mathrm{O}_{3}$ to be the species with the largest dust mass fraction for a star of initial mass $19 M_{\odot}$ their predicted $\mathrm{Al}_{2} \mathrm{O}_{3}$ mass of $0.02 M_{\odot}$ is insufficient to explain the observed far-infrared emission. Instead, sulfides, e.g., $\mathrm{FeS}$ and $\mathrm{Mg}_{0.9} \mathrm{Fe}_{0.1} \mathrm{~S}$, in addition to amorphous carbon and silicates, could potentially contribute to the far-infrared emission.

Within the ejecta of a core-collapse supernova it should be feasible to form a mixture of different dust species. The immediate progenitor star will have been composed of multiple layers of different elements, resulting from the sequence of nuclear reactions. One layer has more carbon than oxygen atoms, while a silicon-rich layer also contains oxygen and iron (e.g., Rauscher et al. 2002). It is believed that different layers are largely unmixed after the SN explosion. Consequently, amorphous carbon dust and silicates can be formed from material originating from the different layers. Given that multiple types of silicates have been inferred to be present in the Galactic supernova remnant Cassiopeia A (Rho et al. 2008; Arendt et al. 2014) and that chemical models predict multiple dust species (Sarangi \& 
Cherchneff 2013), it seems plausible that SN 1987A will have a mixture of different dust species, including both carbonaceous and silicaceous grains.

The mid-infrared observations of SN 1987A at early times $(<1000$ days $)$ implied much smaller ejecta dust masses than derived from the Herschel measurements, e.g., Wooden et al. (1993) estimated a dust mass of $\sim 10^{-4} M_{\odot}$, and later radiative transfer analyses have confirmed that there was $<$ few $\times 10^{-3} M_{\odot}$ of dust at those epochs (Ercolano et al. 2007; Wesson et al. 2015). The large difference between the ejecta dust masses measured then and now implies that the dust mass must have increased significantly over the last $20 \mathrm{yr}$.

The processes to form such a large dust mass over $20 \mathrm{yr}$ may involve dust grain growth. For overall $\mathrm{C} / \mathrm{O}$ number ratios of less than unity, the chemical models of Sarangi \& Cherchneff (2013) predict that carbon atoms should be primarily locked up in CO molecules at early times ( $<1000$ days), preventing the formation of a large mass of amorphous carbon (at most $\left.5.5 \times 10^{-3} M_{\odot}\right)$. Clayton (2011) suggested that a possible solution to forming amorphous carbon is the dissociation of $\mathrm{CO}$ by energetic electrons created by Compton scattering of $\gamma$-rays from radioactive decays. As the ejecta expands and the gas density reduces, the shielding of electrons could decrease, potentially making $\mathrm{CO}$ dissociation more efficient. Additionally, a small fraction of the X-ray radiation from the ring (Helder et al. 2013) could potentially penetrate into the clumpy ejecta, dissociating CO (Hollenbach \& Tielens 1997). The dissociation rate of $\mathrm{CO}$ depends heavily on the gas density and extinction, increasing at lower densities. While the ejecta expands, the gas density decreases, thus the $\mathrm{CO}$ dissociation rate could have increased with time. If atoms or molecules accrete onto existing dust grains, the total dust mass should increase with time.

The supernova explosion produced radioactive isotopes, and the energy generated by their decay should serve as the main heating source of the ejecta. ${ }^{44} \mathrm{Ti}$ is predicted to have become the main radioactive heating source several years after the explosion (Thielemann et al. 1990). The deposited energy from ${ }^{44} \mathrm{Ti}$, extrapolated from Jerkstrand et al. (2011), is estimated to have been $412 L_{\odot}$ in 2012 . This is sufficient to heat the ejecta dust grains, which have a total luminosity of $230 L_{\odot}$. In the SN system, X-ray radiation from the ring can provide a large luminosity of $\sim 500 L_{\odot}$ (Sturm et al. 2010). However, since as seen from the equatorial ring, the ejecta core occupies only about $5 \%$ of the sky, only a small fraction of this luminosity (about $\sim 50 L_{\odot}$ ) may reach the ejecta. So currently ${ }^{44} \mathrm{Ti}$ should be the main dust heating source. The temperature of the dust in the ejecta of SN 1987A is found to be confined to a small range, and this may also support ${ }^{44} \mathrm{Ti}$ as the main heating source for the dust in the ejecta. The expected diffuse distribution of ${ }^{44} \mathrm{Ti}$ within the ejecta seems more likely to heat the dust grains relatively uniformly, whereas external X-ray heating should produce a dust grain temperature gradient within the ejecta.

That the temperature of dust emission from SN 1987A can be fitted by a single temperature component is a significant difference from the Crab Nebula, an older supernova remnant, which shows a broader range of dust temperatures (Gomez et al. 2012). This is most likely caused by the different heating sources and distributions of dust with respect to the heating sources. While the main heating source in the ejecta of SN 1987A is ${ }^{44} \mathrm{Ti}$ decays, the main dust heating source in the Crab Nebula is synchrotron radiation from its pulsar wind nebula (Temim \& Dwek 2013).

\section{CONCLUSIONS}

We have presented dedicated Herschel observations of SN 1987A, taken in 2012. Our photometric and spectroscopic observations confirm that its far-infrared emission is dominated by thermal dust emission from the ejecta, with a minimal contribution from lines, confirming previous analyses that a large mass of dust has formed in the ejecta after the explosion. If we consider predicted elemental mass limits, we would conclude that it is most likely to have formed $0.8 M_{\odot}$ dust in the ejecta, consisting of a combination of $0.5 M_{\odot}$ of silicates and $0.3 M_{\odot}$ of amorphous carbon. If the currently predicted nucleosynthetic limit of $\sim 0.25 M_{\odot}$ of carbon is ignored, then the minimum dust mass that can fit the observed SED is $0.5 M_{\odot}$ of amorphous carbon.

It will be interesting to monitor the ejecta dust in the future, when the main ejecta plunges into the circumstellar ring. As the ejecta expand, the ejecta dust should interact with reverse shocks, with the potential destruction of dust grains (e.g., Jones et al. 1994; Nozawa et al. 2006; Silvia et al. 2012). The efficiency of destruction depends on the position angle of the ejecta with respect to the ring. If most of the ejecta dust can survive future reverse shocks, and future ejecta-ambient ISM shocks, then CCSNe such as SN 1987A may provide a major source of the dust found in the ISMs of galaxies (e.g., Matsuura et al. 2009; Dwek \& Cherchneff 2011; Rowlands et al. 2014).

M.M. acknowledges support from the UK STFC (ST/J001511/1). R.J.I. and L. Dunne acknowledge support from the European Research Council in the form of Advanced Grant COSMICISM. P.v.H. acknowledges support from the Belgian Science Policy Office through the ESA PRODEX program.

\section{REFERENCES}

Arendt, R. G., Dwek, E., Kober, G., Rho, J., \& Hwang, U. 2014, ApJ, 786, 55 Barlow, M. J., Krause, O., Swinbank, B. M., et al. 2010, A\&A, 518, L138 Begemann, B., Dorschner, J., Henning, T., et al. 1997, ApJ, 476, 199 Begemann, B., Dorschner, J., Henning, T., Mutschke, H., \& Thamm, E. 1994, ApJ, 423, L71

Bendo, G. J., Griffin, M. J., Bock, J. J., et al. 2013, MNRAS, 433, 3062

Bouchet, P., Danziger, I. J., \& Lucy, L. B. 1991, AJ, 102, 1135

Bouchet, P., Dwek, E., Danziger, J., et al. 2006, ApJ, 650, 212

Clayton, D. D. 2011, NewAR, 55, 155

Diolaiti, E., Bendinelli, O., Bonaccini, D., et al. 2000, A\&AS, 147, 335

Draine, B. T., \& Lee, H. M. 1984, ApJ, 285, 89

Draine, B. T., \& Li, A. 2007, ApJ, 657, 810

Dwek, E., Arendt, R. G., Bouchet, P., et al. 2010, ApJ, 722, 425

Dwek, E., \& Cherchneff, I. 2011, ApJ, 727, 63

Ercolano, B., Barlow, M. J., \& Sugerman, B. E. K. 2007, MNRAS, 375, 753

Gail, H.-P., \& Sedlmayr, E. 2014, Physics and Chemistry of Circumstellar Dust Shells (Cambridge: Cambridge Univ. Press)

Gall, C., Hjorth, J., \& Andersen, A. C. 2011, A\&ARv, 19, 43

Gomez, H. L., Krause, O., Barlow, M. J., et al. 2012, ApJ, 760, 96

Griffin, M. J., Abergel, A., Abreu, A., et al. 2010, A\&A, 518, L3

Griffin, M. J., North, C. E., Schulz, B., et al. 2013, MNRAS, 434, 992

Groenewegen, M. A. T., Waelkens, C., Barlow, M. J., et al. 2011, A\&A, 526,162

Groningsson, P., Fransson, C., Lundqvist, P., et al. 2008, A\&A, 479, 761

Helder, E. A., Broos, P. S., Dewey, D., et al. 2013, ApJ, 764, 11

Hester, J. J. 2008, ARA\&A, 46, 127

Hildebrand, R. H. 1983, QJRAS, 24, 267

Hildebrand, R. H., \& Dragovan, M. 1995, ApJ, 450, 663

Hollenbach, D. J., \& Tielens, A. G. G. M. 1997, ARA\&A, 35, 179

Hoyle, F., \& Wickramasinghe, N. C. 1991, The Theory of Cosmic Grains (Dordretch: Kluwer)

Indebetouw, R., Matsuura, M., Dwek, E., et al. 2014, ApJL, 782, L2

Jäger, C., Dorschner, J., Mutschke, H., Posch, T., \& Henning, T. 2003, A\&A, 408, 193

Jerkstrand, A., Fransson, C., \& Kozma, C. 2011, A\&A, 530, A45 
Jones, A. P., Tielens, A. G. G. M., Hollenbach, D. J., \& McKee, C. F. 1994, ApJ, 433, 797

Kamenetzky, J., McCray, R. A., Indebetouw, R., et al. 2013, ApJL, 773, L34

Kjær, K., Leibundgut, B., Fransson, C., Jerkstrand, A., \& Spyromilio, J. 2010, A\&A, 517, A51

Kozma, C., \& Fransson, C. 1998, ApJ, 496, 946

Kunkel, W., Madore, B., Shelton, I., et al. 1987, IAU Circ., 4316, 1

Lakićević, M., van Loon, J. T., Stanke, T., De Breuck, C., \& Patat, F. 2012, A\&A, $541, \mathrm{~L} 2$

MacAlpine, G. M., \& Satterfield, T. J. 2008, AJ, 136, 2152

Matsuura, M., Barlow, M. J., Zijlstra, A. A., et al. 2009, MNRAS, 396, 918

Matsuura, M., Dwek, E., Meixner, M., et al. 2011, Sci, 333, 1258

Matsuura, M., Yamamura, I., Cami, J., Onaka, T., \& Murakami, H. 2002, A\&A, 383,972

Meixner, M., Panuzzo, P., Roman-Duval, J., et al. 2013, AJ, 146, 62

Nomoto, K., Tominaga, N., Umeda, H., Kobayashi, C., \& Maeda, K. 2006, NuPhA, 777, 424

Nozawa, T., Kozasa, T., \& Habe, A. 2006, ApJ, 648, 435

Osterbrock, D. E. 1989, Astrophysics of Gaseous Nebulae and Active Galactic Nuclei (Millvalley, CA: Univ. Science Books)

Pilbratt, G. L., Riedinger, J. R., Passvogel, T., et al. 2010, A\&A, 518, L1

Poglitsch, A., Waelkens, C., Geis, N., et al. 2010, A\&A, 518, L2

Rauscher, T., Heger, A., Hoffman, R. D., \& Woosley, S. E. 2002, ApJ, 576,323
Rho, J., Kozasa, T., Reach, W. T., et al. 2008, ApJ, 673, 271

Roche, P. F., Aitken, D. K., \& Smith, C. H. 1991, MNRAS, 252, 39

Rowlands, K., Gomez, H. L., Dunne, L., et al. 2014, MNRAS, 441, 1040

Sarangi, A., \& Cherchneff, I. 2013, ApJ, 776, 107

Semenov, D., Henning, T., Helling, C., Ilgner, M., \& Sedlmayr, E. 2003, A\&A, 410,611

Silvia, D. W., Smith, B. D., \& Shull, J. M. 2012, ApJ, 748, 12

Smith, N. 2013, MNRAS, 434, 102

Staveley-Smith, L., Potter, T. M., Zanardo, G., Gaensler, B. M., \& Ng, C.-Y. 2014, in IAU Symp. 296, Supernova Environmental Impacts, ed. A. Ray \& R. A. McCray (Cambridge: Cambridge Univ. Press), 15

Sturm, R., Haberl, F., Aschenbach, B., \& Hasinger, G. 2010, A\&A, 515, 5

Temim, T., \& Dwek, E. 2013, ApJ, 774, 8

Thielemann, F.-K. K., Hashimoto, M.-A., \& Nomoto, K. 1990, ApJ, 349, 222

Thielemann, F.-K. K., Nomoto, K., \& Hashimoto, M.-A. 1996, ApJ, 460, 408

Weingartner, J. C., \& Draine, B. T. 2001, ApJ, 548, 296

Wesson, R., Barlow, M. J., Matsuura, M., \& Ercolano, B. 2015, MNRAS, 446, 2089

Wooden, D. H., Rank, D. M., Bregman, J. D., et al. 1993, ApJS, 88, 477

Woosley, S. E., \& Weaver, T. A. 1995, ApJS, 101, 181

Zanardo, G., Staveley-Smith, L., \& Indebetouw, R. 2014, ApJ, 796, 82

Zanardo, G., Staveley-Smith, L., Ball, L., et al. 2010, ApJ, 710, 1515

Zubko, V. G., Dwek, E., \& Arendt, R. G. 2004, ApJS, 152, 211

Zubko, V. G., Mennella, V., Colangeli, L., \& Bussoletti, E. 1996, MNRAS, 282,1321 